\title{
Long-term clinical outcome for patients poisoned by the fungal nephrotoxin orellanine
}

\author{
Heidi Hedman ${ }^{1}$, Johan Holmdahl ${ }^{3}$, Johan Mölne ${ }^{4}$, Kerstin Ebefors ${ }^{2}$, Börje Haraldsson ${ }^{1}$ and Jenny Nyström² ${ }^{2 *}$
}

\begin{abstract}
Background: Accidental intake of mushrooms of the Cortinarius species (deadly webcap) may cause irreversible renal damage and the need for dialysis or transplantation. The species is found in forests of Northern Europe, Scandinavia and North America and may be mistaken for other edible mushrooms. The highly selective nephrotoxic compound of the mushroom is called orellanine. Very little is known about the long-term effects of the nephrotoxin.

Methods: We identified patients who ingested deadly webcap in the period of 1979 to 2012. Informed consent and medical records were obtained for 28 of the 39 cases that occurred during the 34-year period. A case control group was also studied based on sex, age and initiation of dialysis or transplantation.

Results: The average age at time of the accidental intake was $40 \pm 3(n=28)$ years. $64 \%$ of patients were male, and 22 of 28 patients developed acute kidney injury requiring dialysis. Serum creatinine peaked at $1329 \pm 133 \mu \mathrm{mol} / \mathrm{l}$, and serum urea was $31 \pm 3.5 \mathrm{mmol} / \mathrm{l}$. No signs of acute damage were present in any other organ. The average time of follow-up was $16.9 \pm 2.1$ years $(1.24-34.3$ years, $n=28) .15$ patients were transplanted and 3 also had a second graft. At follow-up, 23 patients were alive, and five had died at ages of $67 \pm 5$ (range 54-84). The outcome was similar in the case control group with 6 deaths in 20 patients.
\end{abstract}

Conclusion: We conclude that the long-term prognosis for patients poisoned by deadly webcap who lost their renal function is not different compared to other patients in active uremic care.

\section{Background}

The mushroom known as the deadly webcap or Cortinarius rubellus (synonymous with Cortinarius speciosissimus) is occasionally mistaken for eatable chanterelles [1-5], Fig. 1. The habitats are unevenly distributed in Europe and North America and reports of poisoning episodes have been made from forestry areas throughout these regions. One to three of the deadly webcaps is considered enough to cause severe renal failure. There are usually no acute symptoms and the patient will seek medical advice 3 days to a week later with the symptomatology of uraemia caused by an acute kidney injury. The main symptoms of the uraemia at this stage are nausea and fatigue. Edema is less common due

\footnotetext{
* Correspondence: jenny.nystrom@neuro.gu.se

${ }^{2}$ Department of Physiology, Institute of Neuroscience and Physiology, the Sahlgrenska Academy, University of Gothenburg, PO Box 432SE-40530 Gothenburg, Sweden

Full list of author information is available at the end of the article
}

to an initial polyuric phase. This is in turn due to the tubulo-interstitial nephritis caused by damage to the tubular cells. In case of more severe poisoning, the patient becomes anuric after around 1 week [4].

The deadly webcap contains a highly selective nephrotoxin, orellanine, first described by Grzymala [6]. Orellanine (or [2, 2' -bipyridine]-3, 3',4, 4' -tetrol-1, 1' -dioxide) is present in the mushroom in its basic form as a di-glycoside $[7,8]$. The mechanism of action is that orellanine generates oxygen radicals [9-11] and simultaneously shuts down the oxidative defence, by down-regulating most anti-oxidative enzymes [9]. In rodents, the highly kidney-specific nature of orellanine has been verified. Thus, no acute symptoms have been observed at doses $1-2$ orders of magnitude higher than the dose required to elicit acute renal failure $[12,13]$.

There are several case reports of the acute intoxication $[1,2,14-18]$. Due to the lag-phase between intake and symptoms, there are no signs of toxins in blood [19]. 


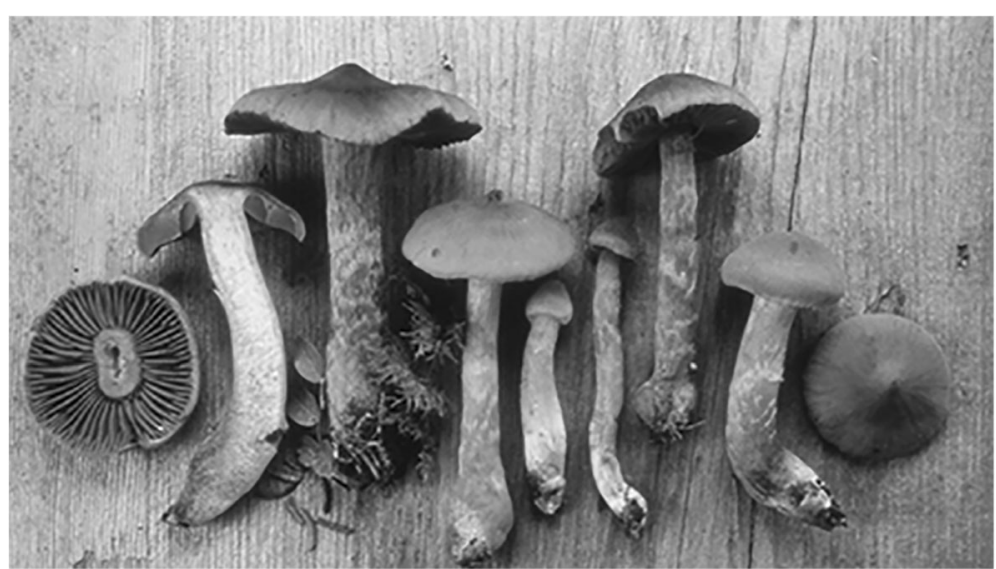

Fig. 1 Specimens of the deadly webcap or Cortinarius rubellus (synonymous with Cortinarius speciosissimus), younger and older mushrooms with slightly different appearance. Picture by Hans Marklund

Suggested therapies during the years have been hemodialysis, hemoperfusion, corticosteroids and antioxidant therapies with highly varying and inconclusive results [4, 20, 21]. Regarding long-term effects of the intoxication, there are only a few reports following transplantation $[17,22]$. There are no long-term follow up on patients after the initial acute kidney injury phase. Therefore, we tried to identify all cases of deadly webcap intoxication during the last three decades in the most affected (South-Western) region of Sweden and compared their outcome with a case-control group of patients. Our hypothesis was that the poison might have long-term effects or increase patient morbidity and mortality. Hence, a long time follow up of a patient cohort would be of interest.

\section{Methods}

\section{Study population}

We included all patients accidentally poisoned by intake of the mushroom deadly webcap (or Cortinarius rubellus, also known as Cortinarius speciosissimus) admitted to any of the hospitals in the Western part of Sweden during the period of January 1979 to December 2012. The total number of patients was 39. In this report, 28 patients were included based on informed consent and available medical records, see Table 1 for demographic data.

\section{Case control population}

For each patient included in the study population that developed CKD5 (chronic kidney disease stage 5), we randomly included another patient as case control from the dialysis unit and another from the transplantation centre at Sahlgrenska University Hospital. Patients with diabetes or other systemic disease were excluded. The case controls were matched in age, sex, comorbidity and the year of the initiation of dialysis or transplantation.
Such case control subjects could be found for all study patients in the dialysis group. However, three case controls are missing during certain years for the transplantation group due to large age differences. Most of the case control patients included had interstitial nephritis (75\%), glomerulonephritis, or polycystic kidney disease, see Table 3 for demographic data.

\section{Kidney morphology}

Kidney biopsies were taken using gauge 16 needles, fixed in buffered paraformaldehyde, embedded in paraffin, sectioned and stained with standard dyes (haematoxylineosin, trichrome, silver and periodic acid-Schiff) and evaluated by a renal pathologist.

\section{Statistical analysis}

Results are presented as mean \pm SEM (standard error of the mean). Differences between groups were calculated using the Gehan-Breslow-Wilcoxon test.

\section{Results \\ Study population}

Of the 39 patients identified, we were able to include 28 patients in the study. This was mainly due to difficulties to be able to get in contact and obtain an informed consent form from all patients.

Of the 28 patients, 22 required dialysis initially, and 6 did not, Table 1 . After 1 month, 21 patients had CKD5 requiring dialysis or transplantation and 7 patients had CKD stage 3 . Thus, there was a $4.5 \%$ chance for a patient initially requiring dialysis to regain renal function during the first month after the intoxication (1/22). Of the 7 patients that were dialysis-free 1 month after the intoxication, all regained most of their renal function within the first year reaching CKD stage 1 or 2 . 


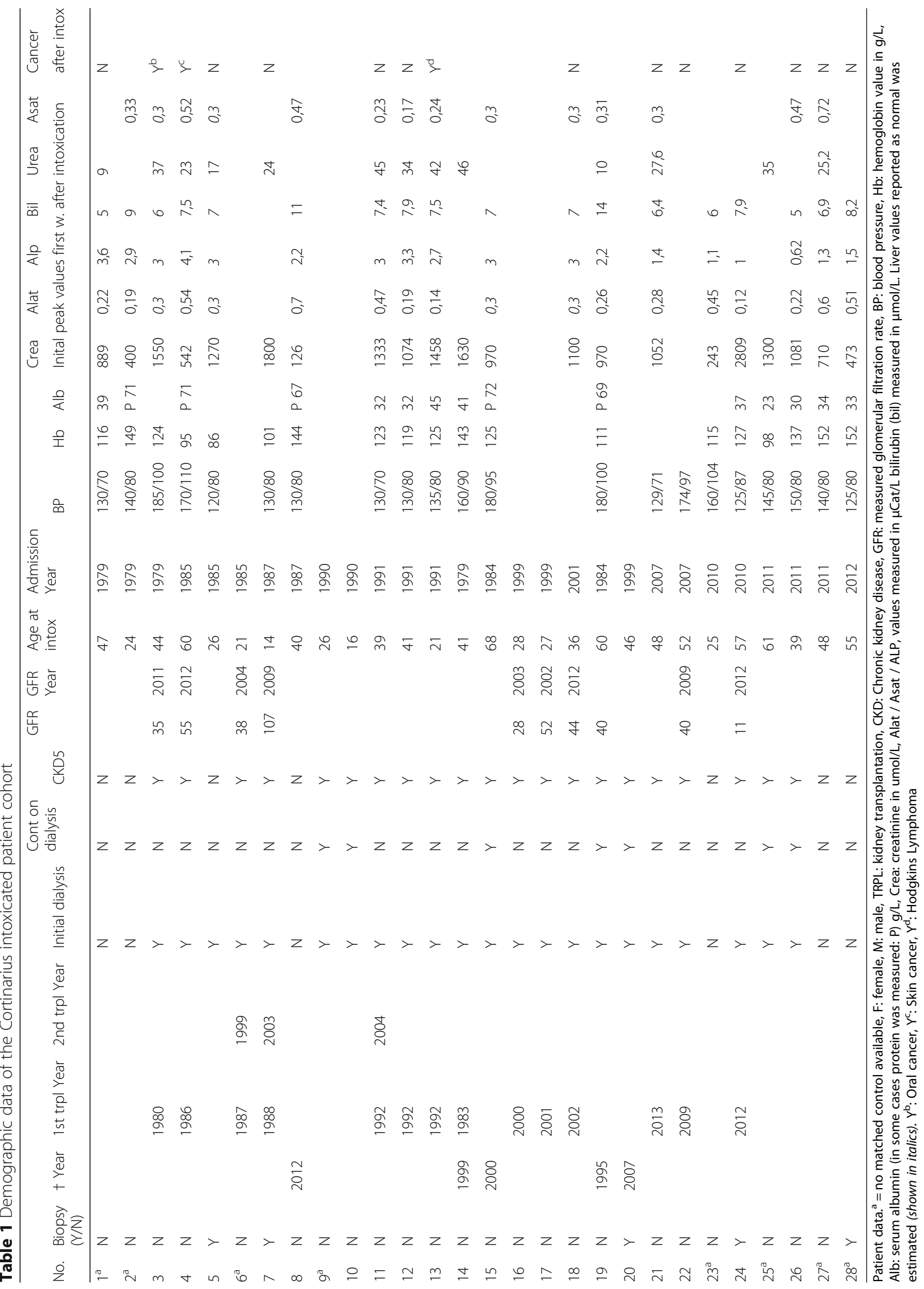


For the total study population of 28 patients, the average time of follow-up was $16.9 \pm 2.1$ years (minimum 1.24 and maximum 34.3 years). 23 patients were alive at the time of follow up, but five had died at ages of $67.0 \pm$ 5.1 years. Over the long observation period, three patients with end stage renal disease (ESRD) and hemodialysis died at the age of $70 \pm 9$ years after $11.9 \pm$ 2.5 years on dialysis.

One patient who never required dialysis died 24 years after the intoxication at the age of 65 . Another patient died 19.1 years after the intoxication and 16 years after a successful kidney transplant at the age of 61 . Fourteen patients were transplanted and of these three had a second kidney graft $12-15$ years after the first one. The average age at the time of the accidental intake of poisonous mushrooms was $40 \pm$ 3 years $(n=28)$, and $64 \%$ of patients were male. In the study group, 3 of the 16 transplanted patients developed cancer in the form of lymphoma, oral or skin cancer. Information of the specific cancer diagnosis was not available for the case control group.

\section{Acute effects}

The serum creatinine of the patients requiring dialysis peaked at $1329 \pm 133 \mu \mathrm{mol} / \mathrm{l}(n=15)$ and serum urea reached $31 \pm 3.5 \mathrm{mmol} / \mathrm{l}(n=11)$. The peak creatinine and urea values were markedly lower in patients that did not require dialysis, Table 1 . Laboratory tests did not show signs of damage in any other organ apart from the kidney. Thus, the aspartate amino transferase (ASAT), alanine amino transferase (ALAT), alkaline phosphatase (ALP) and bilirubin values were all within their normal limits in the patients analyzed; $0.35 \pm 0,04 \mu \mathrm{Cat} / \mathrm{l}, 0.34 \pm$ $0.04 \mu \mathrm{Cat} / \mathrm{l}, 2.38 \pm 0.04 \mu \mathrm{Cat} / \mathrm{l}$, and $7.59 \pm 0.5 \mu \mathrm{mol} / \mathrm{l}$ respectively $\left(\mathrm{n}_{\mathrm{ALAT}}=14, \mathrm{n}_{\mathrm{ASAT}}\right.$, ALP, bilirubin $\left.=18\right)$.

\section{Patients with non-dialysis dependent kidney injury}

Six of the patients that were orellanine intoxicated developed a less severe kidney injury and never required dialysis. On average their serum creatinine peaked at $474 \pm 117 \mu \mathrm{mol} / \mathrm{l}(n=3)$ during the first week. Initially, two patients were treated with three sessions each of hemoperfusion with charcoal, which in 1979 was suggested as therapy. One patient initially required dialysis and had a serum creatinine of $1270 \mu \mathrm{mol} / \mathrm{l}$, but regained part of her renal function within the first month, and had a glomerular filtration rate of $87 \mathrm{ml} / \mathrm{min} 1$ year later determined by ${ }^{51} \mathrm{Cr}$-EDTA clearance. The 7 patients who did not develop CKD5 were followed over a timeperiod of $18.3 \pm 5.8$ years, range $1.2-34.3$ years.

Patients with dialysis-dependent chronic kidney disease stage 5 One month after the intoxication, 21 of 22 patients still required dialysis. 14 of them were transplanted, 6 received a kidney graft from a living donor and 8 from a dead donor. Before transplantation, the patients had been on dialysis for $19 \pm 4$ months (range 7-64 months). Three renal grafts lost their function after 11, 12 and 15 years and a second kidney transplantation was performed. Those patients now have been living with their 2 nd grafts for 10, 11 and 15 years, Table 1 .

\section{Kidney morphology}

Kidney biopsies were obtained from 5/28 patients. The biopsy material from one patient could not be retrieved and thus material was available from 4 patients. According to clinical data biopsies were obtained 10-21 days after fungal ingestion. All biopsies showed a similar pattern dominated by acute tubular necrosis (ATN), Fig. 2. Two patients (no 5 and 28) showed less severe damage with focal involvement, while the other two biopsies (no 20 and 24) showed a diffuse involvement with generalized inflammation and tubular damage. These biopsies also showed tubular dilatation, tubular cellular atrophy and degeneration, Table 2. All biopsies contained some apoptotic bodies, interstitial edema and focal inflammation but no eosinophilia. Fibrosis was not a prominent finding.

\section{Case control population}

The case control patients were age and sex matched, and they started dialysis or were transplanted in the same year as the patients in the study population, Table 3. No acceptable case control could be found for three of the 16 transplanted study patients since the candidates were either too old or had systemic disease. The distribution of kidney disease within the population is displayed in Table 3. In total, 20 patients were matched,

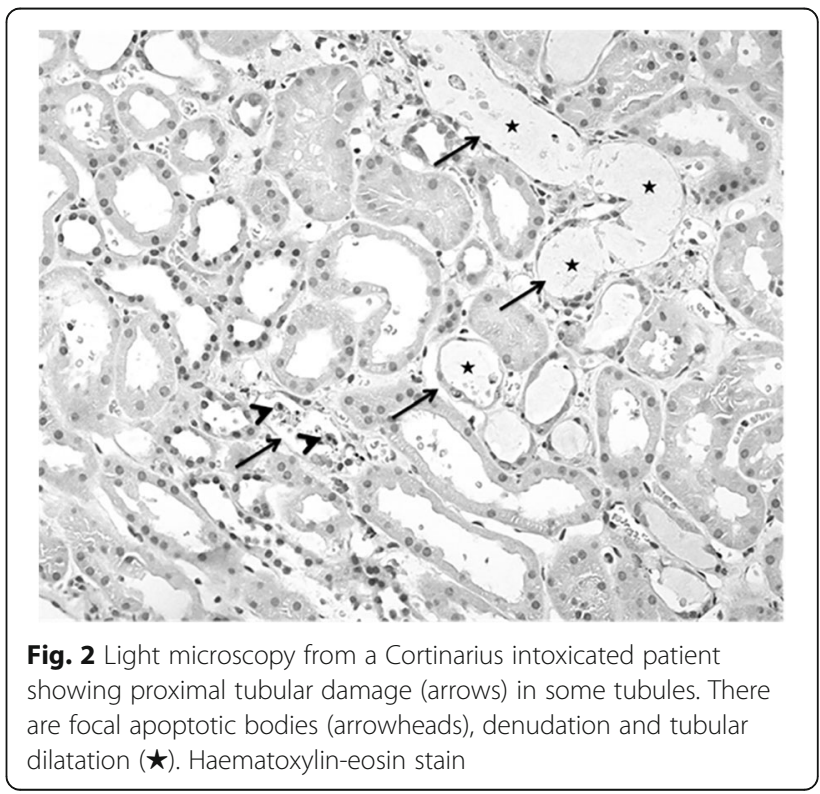


Table 2 Renal morphology of biopsies from patients with Cortinarius poisoning

\begin{tabular}{|c|c|c|c|c|c|c|}
\hline \multirow{2}{*}{$\begin{array}{l}\text { Patient No. } \\
5\end{array}$} & \multirow{2}{*}{$\begin{array}{l}\text { Time after ingestion } \\
2 v\end{array}$} & \multicolumn{2}{|c|}{$\begin{array}{l}\text { Tubular damage: Necrosis, } \\
\text { apoptosis, degeneration, } \\
\text { dilatation, involvement }\end{array}$} & \multirow{2}{*}{$\begin{array}{l}\text { Edema } \\
\text { minimal }\end{array}$} & \multirow{2}{*}{$\begin{array}{l}\text { Inflammation } \\
\text { minimal }\end{array}$} & \multirow{2}{*}{$\begin{array}{l}\text { Fibrosis } \\
\text { minimal }\end{array}$} \\
\hline & & $0+00$ & focal & & & \\
\hline 7 & - & - & & - & - & - \\
\hline 20 & $3 v$ & ++++ & diffuse & severe & prominent & minimal \\
\hline 24 & $>10 d$ & $0+++$ & diffuse & prominent & slight & slight \\
\hline 28 & 10d & $0+00$ & focal & focal & minimal & minimal \\
\hline
\end{tabular}

7 of the patients were on dialysis and the remaining 13 patients were transplanted, one of them twice. Of the 20 patients, 6 died at a mean age of $67.5 \pm 4.7$ years after $16.9 \pm 6.6$ years on dialysis $(n=3$, at a mean age of 68 years) and $10.3 \pm 2.2$ years after transplantation $(n=3$, at a mean age of 66 year), Fig. 3. In the case control population, one patient died of cancer and three other patients developed tumours during the observation period.

\section{Comparisons between study and control populations}

Of the 21 patients with ESRD in the study group, 20 were matched; 7 in dialysis and 13 that were transplanted. Cancer was not more frequent in the study group compared to control group. There were 3 cases of cancer in the study population of 21 patients (14\%), of which all 3 were transplanted. In the control group 3 out of $20(15 \%)$ were diagnosed with cancer, two of which were transplanted and one which remained in

Table 3 The table describes the demographic data available for the matched control cohort ${ }^{\mathrm{a}}$ second transplant in 2003

\begin{tabular}{|c|c|c|c|c|c|c|c|c|}
\hline \multicolumn{9}{|c|}{ A. Matched control patients who had hemodialysis } \\
\hline No\#: & Born year & Transplant 1 & Diagnosis & Follow up M & Follow up Y & Death $Y$ & CoD & Cancer \\
\hline 1 & 1935 & 1980 & PCK & 356 & 30 & 2010 & Peritonitis & Y \\
\hline 2 & 1925 & 1986 & Amylioidosis & 95 & 8 & 1994 & Unknown & Y \\
\hline 3 & 1965 & 2002 & Crescentic GN & 141 & 12 & & & N \\
\hline 4 & 1953 & 2012 & $\lg A N$ & 20 & 2 & & & N \\
\hline 5 & 1958 & 2012 & PCK & 22 & 2 & & & N \\
\hline 6 & 1955 & 2009 & PCK & 59 & 5 & & & N \\
\hline 7 & 1942 & 1983 & Pyelonephritis & 368 & 31 & & & \\
\hline 8 & 1972 & $1988^{\mathrm{a}}$ & PCK & 307 & 26 & & & N \\
\hline 9 & 1970 & 2000 & Interstitial Nephritis & 156 & 13 & & & \\
\hline 10 & 1973 & 2001 & $\lg A N$ & 194 & 16 & & & \\
\hline 11 & 1952 & 1992 & $\lg A N$ & 157 & 13 & 2005 & Epilepsy & N \\
\hline 12 & 1951 & 1992 & Interstitial nephritis & 263 & 22 & & & $\mathrm{~N}$ \\
\hline 13 & 1971 & 1992 & CGN & 260 & 22 & & & \\
\hline \multicolumn{9}{|c|}{ B. Matched control patients who had a renal transplantation } \\
\hline No\#: & Born year & HD start & Diagnosis & Follow up M & Follow up Y & Death $Y$ & CoD & Cancer \\
\hline 14 & 1925 & 1984 & CVD & 160 & 13 & 1997 & Cancer & Y \\
\hline 15 & 1916 & 1984 & Heredetary nephropaty & 139 & 12 & 1996 & Sepsis & \\
\hline 16 & 1962 & 1985 & Glomerulonephritis & 343 & 29 & & & Y \\
\hline 17 & 1942 & 1987 & CVD & 72 & 6 & 1993 & CVD & \\
\hline 18 & 1965 & 1990 & Glomerulonephritis & 284 & 24 & & & \\
\hline 19 & 1952 & 1999 & Glomerulonephritis & 178 & 15 & & & \\
\hline 20 & 1973 & 2011 & Interstitial nephritis & 29 & 2 & & & $\mathrm{~N}$ \\
\hline
\end{tabular}

${ }^{a}$ Second transplant in 2003, M: months, Y: year, PCK:polycystic kidney disease, Crescentic GN: crescentic glomerulonephritis, IgAN: IgA nephritis, CGN: Chronic glomerulonephritis, COD: cause of death, CVD: cardiovascular disease

The cohort was matched based on age, sex and A. initiation of hemodialysis or B. renal transplantation 


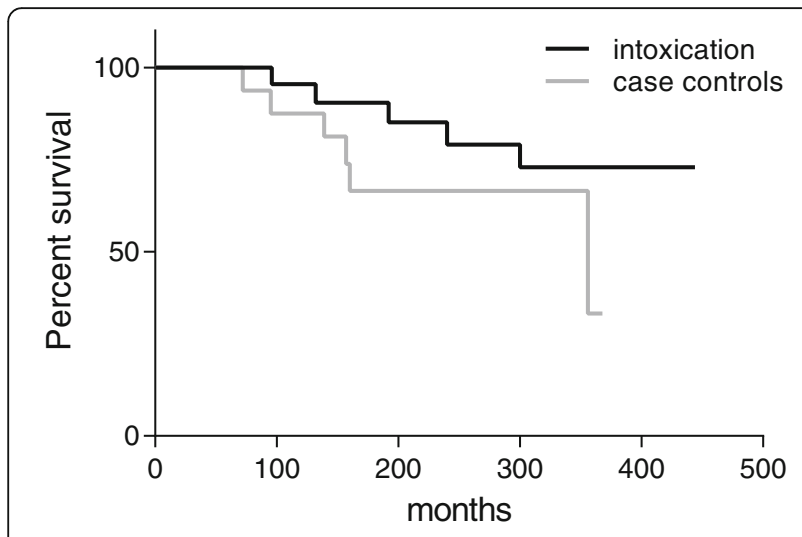

Fig. 3 Kaplan Mayer survival curve over the two groups. No statistical differences were seen between the study group (intoxicated patients) and the control group over time

dialysis. In total, 4 patients of 21 with ESRD died in the study group compared to 6 in the control group, see Fig. 3. There was no statistical difference between survivals in the study group compared to the case control group.

\section{Discussion}

The present study was done to reveal if patients that accidentally ingested deadly webcap (Cortinarius rubellus) suffered from long-term increases in morbidity or mortality. Indeed, $75 \%$ of the 28 patients developed CKD5, and $70 \%$ of them were transplanted. However, we did not find evidence of any other damage apart from the severe kidney injury. It should be noted that this is still a small patient group and of 39 cases we were only able to investigate 28 cases. Possible confounders could be the limited size of the study or that bias was introduced by co-morbidities or other medication. However, we do not anticipate that this has affected the outcome of the study.

The follow up period after the accidental intake of poisonous webcap is quite long, 16.9 years, with a range of up to 34.3 years. As expected, the long-term survival was excellent after transplantation as reflected by the current follow up period of $19.0 \pm 2.5$ years for the 14 patients in that group. The result is in accordance with previous reports over shorter periods of follow up [17, 22]. Regarding dialysis, it is well known that the mortality is high for all age groups [23]. Naturally, the survival on dialysis will depend, not only on the dialysis practice, but also on the underlying disease and on comorbidities [23-25]. The outcome was quite good for the study population on dialysis, which so far has been followed for $12.4 \pm 3.4$ years. Some patients have died over the three and a half decades of follow up. However, the patients on dialysis died after more than 12 years of treatment with haemodialysis at a mean age of 68 . A similar picture was found in the case control population with follow up periods for transplanted patients of $15.2 \pm$ 2.8 years and of patients on dialysis for $14.4 \pm 3.5$ years. In the case control population there were three deaths in each of the two ESRD groups, with transplanted patients being 66 years at the time of death, and patients on dialysis being 68 . We found no evidence of increased risk for malignancy, endocrinological disorders or other morbidity in the study population.

\section{Conclusion}

We conclude that the long-term outcome is equally good for patients that have lost their renal function due to accidental intake of deadly webcap compared to other reasons for the uraemia. This was true both for transplanted patients and patients treated with dialysis.

\section{Abbreviations \\ ${ }^{51} \mathrm{Cr}$-EDTA: 51 Chromium-Ethylenediaminetetraacetic acid; ALAT: Alanine amino transferase; ALP: Alkaline phosphatase; ASAT: Aspartate amino transferase; ATN: Acute tubular necrosis; CKD5: Chronic kidney disease stage 5; ESRD: End stage renal disease; SEM: Standard error of the mean}

\section{Acknowledgements}

We thank Lena Heijdenberg for excellent assistance with patient data retrieval and Hans Marklund for providing a photo of the deadly webcaps.

\section{Funding}

This study was supported by the Swedish Medical Research council grants (09898, 14764 and 20852), VINNOVA, the National Association for Kidney Disease, the Lars Erik Gelin Memorial Foundation, the IngaBritt and Arne Lundberg Research Foundation and the Sahlgrenska University Hospital grant ALF.

\section{Availability of data and materials}

No data has been submitted to any open access databases. Full patient records are being kept coded.

\section{Authors' contributions}

$\mathrm{BH}$ and $\mathrm{JN}$ outlined the study design. $\mathrm{HH}, J \mathrm{H}, \mathrm{JM}, \mathrm{BH}, \mathrm{JN}$ conducted the study, $\mathrm{JH}$ and $\mathrm{BH}$ contacted and informed the patients and $\mathrm{JM}$ performed immunohistochemistry staining. $\mathrm{HH}, \mathrm{KE}, \mathrm{BH}, \mathrm{JN}$ analysed the data and drafted the manuscript. $\mathrm{HH}, J \mathrm{H}, J \mathrm{JM}, \mathrm{KE}, \mathrm{BH}, \mathrm{JN}$ wrote and finalized the manuscript and provided critical review. All authors read and approved the final manuscript.

\section{Competing interests}

The authors declare that they have no competing interests. $\mathrm{HH}, \mathrm{BH}, \mathrm{JN}$ are in a commercialized project investigating the possibility of using orellanine as a treatment for metastasizing renal clear cell carcinoma. The funders listed in Acknowledgements had no role in study design, data collection and analysis, decisions to publish, or preparation of the manuscript.

\section{Consent for publication}

Not applicable.

\section{Ethics approval and consent to participate}

This study was conducted in accordance with the declaration of Helsinki and with the approval of the local ethical board of West Sweden in Gothenburg. The approval number is 1017-12.

All patients included in the study was informed both orally and in written form and patients who chose to participate signed a written consent form before participating.

\section{Publisher's Note}

Springer Nature remains neutral with regard to jurisdictional claims in published maps and institutional affiliations. 


\section{Author details}

'Department of Molecular and Clinical Medicine, Institute of Medicine, the Sahlgrenska Academy, University of Gothenburg, Gothenburg, Sweden. ${ }^{2}$ Department of Physiology, Institute of Neuroscience and Physiology, the Sahlgrenska Academy, University of Gothenburg, PO Box 432SE-40530 Gothenburg, Sweden. ${ }^{3}$ Department of Nephrology, Institute of Medicine, The Sahlgrenska Academy, University of Gothenburg, Gothenburg, Sweden. ${ }^{4}$ Department of Pathology, Institute of Biomedicine, The Sahlgrenska Academy, University of Gothenburg, Gothenburg, Sweden.

Received: 1 April 2016 Accepted: 24 March 2017

\section{Published online: 03 April 2017}

\section{References}

1. Evans N, Hamilton A, Bello-Villalba MJ, Bingham C. Irreversible renal damage from accidental mushroom poisoning. BMJ. 2012;345:e5262

2. Talmud D, Wynckel A, Grossenbacher F, Saad S, Motte J, Abely M, Pietrement C. Four family cases of acute renal failure. Diagnosis: Orellanus syndrome. Pediatr Nephrol. 2011;26(3):385-8

3. Mount $P$, Sinclair $S$, Finlay M, Harris $G$, Becker $G$. Three cases of acute renal failure secondary to ingestion of wild mushrooms. Nephrology. 2002;7(1):A152

4. Holmdahl J. Mushroom poisoning: Cortinarius speciosissimus nephrotoxicity. Gothenburg: University of Gothenburg; 2001.

5. Grzymala S. Massenvergiftungen durch den orangefuchsigen Hautkopf. Z Pilzkd. 1957:23:139-42.

6. Grzymala S, Fiksinski R. Use of micro-electrofiltration in the precipitation of thermal decomposition products of orellanine in the volatile phase. Postepy Hig Med Dosw. 1960;14:699-702.

7. Herrmann A, Hedman H, Rosen J, Jansson D, Haraldsson B, Hellenas KE. Analysis of the mushroom nephrotoxin orellanine and its glucosides. J Nat Prod. 2012;75(10):1690-6.

8. Spiteller P, Spiteller M, Steglich W. Occurrence of the fungal toxin orellanine as a diglucoside and investigation of its biosynthesis. Angew Chem Int Ed Engl. 2003;42(25):2864-7.

9. Nilsson UA, Nystrom J, Buvall L, Ebefors K, Bjornson-Granqvist A, Holmdahl J, Haraldsson B. The fungal nephrotoxin orellanine simultaneously increases oxidative stress and down-regulates cellular defenses. Free Radic Biol Med. 2008:44(8):1562-9.

10. Oubrahim H, Richard JM, Cantin-Esnault D. Peroxidase-mediated oxidation, a possible pathway for activation of the fungal nephrotoxin orellanine and related compounds. ESR and spin-trapping studies. Free Radic Res. 1998:28(5):497-505.

11. Richard JM, Cantin-Esnault D, Jeunet A. First electron spin resonance evidence for the production of semiquinone and oxygen free radicals from orellanine, a mushroom nephrotoxin. Free Radic Biol Med. 1995;19(4):417-29.

12. Prast H, Werner ER, Pfaller W, Moser M. Toxic properties of the mushroom Cortinarius orellanus. I. Chemical characterization of the main toxin of Cortinarius orellanus (Fries) and Cortinarius speciosissimus (Kuhn \& Romagn) and acute toxicity in mice. Arch Toxicol. 1988;62(1):81-8.

13. Kurnsteiner $\mathrm{H}$, Moser $\mathrm{M}$. Isolation of a lethal toxin from Cortinarius orellanus Fr. Mycopathologia. 1981;74(2):65-72.

14. Frank H, Zilker T, Kirchmair M, Eyer F, Haberl B, Tuerkoglu-Raach G, Wessely $M$, Grone HJ, Heemann U. Acute renal failure by ingestion of Cortinarius species confounded with psychoactive mushrooms: a case series and literature survey. Clin Nephrol. 2009;71(5):557-62.

15. Mount P, Harris G, Sinclair R, Finlay M, Becker GJ. Acute renal failure following ingestion of wild mushrooms. Intern Med J. 2002;32(4):187-90.

16. Horn $\mathrm{S}$, Horina $\mathrm{JH}$, Krejs $\mathrm{G}$, Holzer $\mathrm{H}$, Ratschek $M$. End-stage renal failure from mushroom poisoning with Cortinarius orellanus: report of four cases and review of the literature. Am J Kidney Dis. 1997:30(2):282-6.

17. Holmdahl J, Blohme I. Renal transplantation after Cortinarius speciosissimus poisoning. Nephrol Dial Transplant. 1995;10(10):1920-2.

18. Holmdahl J, Mulec $\mathrm{H}$, Ahlmen J. Acute renal failure after intoxication with Cortinarius mushrooms. Hum Toxicol. 1984;3(4):309-13.

19. Rohrmoser $M$, Kirchmair M, Feifel E, Valli A, Corradini R, Pohanka $E_{i}$ Rosenkranz A, Poder R. Orellanine poisoning: rapid detection of the fungal toxin in renal biopsy material. J Toxicol Clin Toxicol. 1997;35(1):63-6.
20. Wornle M, Angstwurm MW, Sitter T. Treatment of intoxication with Cortinarius speciosissimus using an antioxidant therapy. Am J Kidney Dis. 2004;43(4):e3-6.

21. Fulde K, Bohler J, Keller E, Frahm AW. Efficiency of haemoperfusion materials at removing the fungal toxin orellanine from human plasma. Pharmazie. 1998:53(1):58-9.

22. Nagaraja $P$, Thangavelu A, Nair H, Kumwenda M. Successful living related kidney transplantation for end-stage renal failure caused by orellanine syndrome. QJM 2015;108(5):413-5.

23. Zhang Y, Cotter DJ, Thamer M. The effect of dialysis chains on mortality among patients receiving hemodialysis. Health Serv Res. 2011:46(3):747-67.

24. Stack AG, Neylon AM, Abdalla AA, Hegarty A, Hannigan A, Cronin CJ, Nguyen HT, Casserly LF. Declining mortality rates despite increases in clinical coronary artery disease among US dialysis patients: a national registry study. Am J Nephrol. 2013:38(1):66-74.

25. Foote C, Ninomiya T, Gallagher M, Perkovic V, Cass A, McDonald SP, Jardine M. Survival of elderly dialysis patients is predicted by both patient and practice characteristics. Nephrol Dial Transplant. 2012;27(9):3581-7.

\section{Submit your next manuscript to BioMed Central and we will help you at every step:}

- We accept pre-submission inquiries

- Our selector tool helps you to find the most relevant journal

- We provide round the clock customer support

- Convenient online submission

- Thorough peer review

- Inclusion in PubMed and all major indexing services

- Maximum visibility for your research

Submit your manuscript at www.biomedcentral.com/submit 